\title{
Análise da Custo-Efetividade da Angiotomografia Coronariana no SUS, em Comparação com Outros Métodos Não Invasivos na Suspeita de DAC Estável
}

\author{
Cost-Effectiveness Analysis of CCTA in SUS, as Compared to Other Non-Invasive Imaging Modalities in \\ Suspected Obstructive CAD
}

\author{
Patricia Bastos do Carmo, ${ }^{1 \oplus}$ Carlos Alberto da Silva Magliano, ${ }^{1 \oplus}$ Helena Cramer Veiga Rey, ${ }^{\circledR}$ Gabriel C. \\ Camargo, ${ }^{1}$ Luís Filipe Lannes Trocado, ${ }^{1}$ Ilan Cottlieb ${ }^{1,2}{ }^{\circledR}$ \\ Instituto Nacional de Cardiologia, ${ }^{1}$ Rio de Janeiro, RJ - Brasil \\ Casa de Saúde São José, ${ }^{2}$ Rio de Janeiro, RJ - Brasil
}

\section{Resumo}

Fundamento: Atualmente o sistema de saúde público brasileiro (SUS) não contempla a angiotomografia de coronárias.

Objetivos: Ranquear sob a perspectiva do SUS, a custo-efetividade de estratégias diagnósticas combinando teste ergométrico, cintilografia miocárdica, ecocardiograma por estresse e angiotomografia de coronárias para o diagnóstico de doença arterial coronariana em uma coorte hipotética de pacientes com probabilidade pré-teste intermediária.

Métodos: Análise de custo-efetividade por meio de árvore de decisão. Foram analisados a relação de custo-efetividade incremental e o benefício líquido em saúde das estratégias diagnósticas, com a adoção de múltiplos limiares de disposição a pagar entre 0,05 e 1 PIB per capita por diagnóstico correto. Nos casos de testes sequenciais, um segundo teste confirmatório era realizado quando o primeiro fosse positivo.

Resultados: Após exclusão das estratégias diagnósticas dominadas ou com dominância estendida, a fronteira de eficiência foi composta por três estratégias: teste ergométrico, teste ergométrico seguido de ecocardiograma de estresse, e ecocardiograma de estresse seguido de angiotomografia de coronárias, sendo esta última a estratégia mais custo-efetiva. Pelo critério do benefício líquido, o ranqueamento das estratégias mais custo-efetivas variou conforme a disposição a pagar.

Conclusão: Utilizando conceitos atuais de avaliação de tecnologias em saúde, este estudo fornece um ranqueamento para a tomada de decisão sobre qual estratégia diagnóstica utilizar, em uma população com risco pré-teste intermediário para DAC. Com estimativa factível de custos para a ATC, o impacto da inclusão desta ao rol do arsenal diagnóstico representaria uma estratégia custo-efetiva na maioria dos cenários avaliados nas variações de disposição a pagar.

Palavras-chave: Doença das Coronárias; Angina Estável; Análise Custo-Benefício; Técnicas de Diagnóstico Cardiovascular.

\section{Abstract \\ Background: The Brazilian public health system does not include computed tomography angiography (CTA).}

Objective: Rank, according to the Brazilian public health system, the cost-effectiveness of different strategies for the diagnosis of coronary artery disease (CAD), combining exercise tests (ET), myocardial scintigraphy (MS), stress echocardiography (SE), and CTA in a hypothetical intermediate pre-test probability cohort of patients.

Methods: This study implemented a cost-effectiveness analysis through a decision tree. The incremental cost-effectiveness ratio (ICER) and net benefit were analyzed by adopting multiple thresholds of willingness to pay, from 0.05 to 1 GDP per capita per correct diagnosis. In sequential tests, a second confirmatory test was performed only when the first was positive.

Results: After excluding dominated or extended dominance diagnostic strategies, the efficiency frontier consisted of three strategies: ET, ET followed by SE, and SE followed by CTA, the last being the most cost-effective strategy. Through the net benefit, the ranking of the most costeffective strategies varied according to willingness to pay.

Correspondência: Patricia Bastos do Carmo •

Instituto Nacional de Cardiologia - Rua das Laranjeiras 374. CEP 22240-006, Rio de Janeiro, RJ - Brasil

E-mail: pati_bastos2@hotmail.com

Artigo recebido em 05/10/2020, revisado em 04/03/2021, aceito em 24/03/2021

DOI: https://doi.org/10.36660/abc. 20201050 
Conclusions: Using current concepts of health technology assessment, this study provides a ranking for decision-making concerning which diagnostic strategy to use in a population with an intermediate pre-test risk for CAD. With a feasible cost estimate adopted for CTA, the impact of including this to the list of the diagnostic arsenal would represent a cost-effective strategy in most of the evaluated scenarios with broad variations in the willingness to pay.

Keywords: Coronary Disease; Stable Angina; Cost-Benefit Analysis; Diagnostic Techniques, Cardiovascular.

Full texts in English - http://www.arquivosonline.com.br

\section{Introdução}

A doença cardiovascular foi causa de 17,7 milhões de óbitos em 2015, representando 31\% de todas as mortes em nível global. Desses, estima-se que 7,4 milhões ocorram devido à doença coronariana (DAC). ${ }^{1,2} \mathrm{No}$ Brasil, de acordo com última atualização dos indicadores de saúde, foram registrados aproximadamente 490.000 óbitos por DAC no período de 2007 a 2011. ${ }^{3}$ Estimase que a prevalência de angina leve e angina moderada à grave na população brasileira seja respectivamente de $7,6 \%$ e 4,2\%, ${ }^{4}$ e os custos relacionados a doenças cardiovasculares crescem à medida que a população envelhece, sendo estimado em 2015, para o Brasil, um total de R\$37,1 bilhões de reais, aproximadamente $0,7 \%$ do produto interno bruto (PIB). ${ }^{5}$

A cineangiocoronariografia (CAT) é o "padrão-ouro" para diagnóstico de DAC, porém é exame invasivo e associado a complicações. ${ }^{6,7}$ Idealmente, testes diagnósticos não invasivos deveriam selecionar quais pacientes seriam encaminhados para confirmação diagnóstica invasiva, mas a estratégia atual é falha, como demonstrado em um grande registro de 398.978 pacientes encaminhados à CAT, dos quais apenas $37 \%$ apresentaram DAC obstrutiva, apesar de testes não invasivos terem sido realizados em $84 \%$ dos pacientes (em sua maioria funcionais). ${ }^{8} \mathrm{Um}$ estudo brasileiro corrobora esses achados, no qual $61 \%$ dos pacientes com testes funcionais com critérios de alto risco não apresentaram DAC obstrutiva. ${ }^{9}$ Novos testes diagnósticos com maior acurácia ou estratégias diagnósticas sequenciais têm potencial de reduzir os erros diagnósticos e o número de CAT desnecessárias.

Identificar a estratégia diagnóstica para DAC obstrutiva mais custo-efetiva pode trazer benefícios clínicos e econômicos para o SUS. Hoje, além da CAT, os testes diagnósticos para DAC disponíveis no SUS são: cintilografia miocárdica (CM), ecocardiograma de estresse (ECO) e teste ergométrico (TE). A angiotomografia coronariana (ATC) é um exame ainda não incorporado no SUS, embora apresente alta acurácia diagnóstica, quando comparada aos demais. ${ }^{10-13}$

O objetivo deste estudo é ranquear a custo-efetividade das diferentes estratégias diagnósticas de DAC, considerando os testes não invasivos disponíveis no SUS, e a ATC, testando variados limiares de disposição a pagar, para uma população pré-estabelecida de probabilidade pré-teste intermediária de $30 \%$, dentro da perspectiva do SUS.

\section{Métodos}

A razão de custo-efetividade incremental (RCEI) tem sido rotineiramente utilizada por agências de avaliação de tecnologia em saúde em todo o mundo para resumir os resultados das avaliações econômicas e estabelecer a custo-efetividade das tecnologias. Entretanto, uma nova metodologia de avaliação de custo-efetividade foi proposta: o benefício líquido, que pode ser monetário, em inglês, net monetary benefit (NMB), ou benefício em saúde, em inglês, net health benefit (NHB). ${ }^{14}$ Essa última metodologia tem vantagens sobre a RCEI, por não necessitar de um comparador base para estimativa de ganhos e custos incrementais e por ser mais fácil calcular. A eficácia ou "benefício" de cada estratégia pode ser medida em diferentes formas, como anos de vida salvos, ou pelo número de diagnósticos corretos obtidos com uma estratégia diagnóstica. Estima-se, com base em uma disposição a pagar pré-estabelecida, o "lucro" obtido com a intervenção. Por exemplo, se um decisor está disposto a pagar $\mathrm{R} \$ 10$ mil reais por ano de vida salvo, uma tecnologia que aumentasse em 5 anos a sobrevida "valeria" $\mathrm{R} \$ 50$ mil reais. Caso o preço da intervenção fosse inferior a $\mathrm{R} \$ 50$ mil reais, ela seria benéfica. Para um valor hipotético de $\mathrm{R} \$ 30$ mil reais, tal intervenção estaria fornecendo um NMB de $\mathrm{R} \$ 20$ mil reais $(5 \times \mathrm{R} \$ 10$ mil - R\$ 30 mil). Da mesma maneira, considerando a mesma disposição a pagar, esperamos um ganho mínimo de 3 anos de vida com tal investimento (30 mil/R\$ $10 \mathrm{mil}$ ), mas, como ele fornece 5 anos de sobrevida, teremos um NHB de 2 anos. Quanto maior o ganho monetário ou em saúde, maior a custoefetividade daquela tecnologia ou estratégia diagnóstica, pois sua incorporação trará economia e ganhos em saúde.

A custo-efetividade dos testes diagnósticos para DAC obstrutiva (ATC, CM, ECO e TE) foi avaliada usando uma combinação de 11 estratégias diagnósticas e o impacto sobre uma coorte hipotética de 1.000 indivíduos com 30\% de prevalência de DAC (probabilidade intermediária). Um teste negativo representava o fim da pesquisa. Nos casos em que a estratégia diagnóstica envolvesse testes sequenciais, um segundo teste confirmatório era realizado apenas caso o primeiro teste fosse positivo. A soma de testes verdadeiros negativos (teste negativo em pacientes sem DAC > 50\%) com testes verdadeiros positivos (testes positivos em pacientes com DAC $>50 \%$ ) representou o total de diagnósticos corretos.

Para definição da estratégia mais custo-efetiva, foram adotadas duas análises: a fronteira de eficiência, com base na RCEI e o NHB. Por não haver um limiar de custo-efetividade estabelecido no Brasil, todas as tecnologias não dominadas ou sem dominação estendida foram apresentadas por meio de uma fronteira de eficiência. Com as estratégias ranqueadas de acordo com seus custos ou benefícios, a estratégia dominada será simplesmente aquela menos eficaz e mais cara.

A segunda etapa de análise pelo RCEl envolve a identificação de estratégias com dominância estendida. As estratégias não dominadas foram ranqueadas por ordem crescente de custos, e a RCEI, calculada comparando os custos e efetividade incrementais relativamente à estratégia menos onerosa precedente. As estratégias menos eficazes e com uma RCEI 
mais elevada foram consideradas não custo-efetivas por dominância estendida.

A estratégia mais custo-efetiva, por definição, é aquela que apresenta a maior RCEI, dentro do limiar de disposição a pagar estabelecido pelo tomador de decisão. No caso do $\mathrm{NHB}$, a estratégia mais custo-efetiva será aquela que trouxer o maior ganho líquido em número de diagnósticos corretos, de acordo com cada limiar de disposição a pagar. Para ambas as análises, foi necessário estimar os custos e a efetividade (quantidade de testes corretamente diagnosticados) das diferentes estratégias.

As estratégias mais custo-efetivas foram também ranqueadas de acordo com a variação de probabilidades pré-teste entre $10 \%$ e $60 \%$ em diferentes limiares de disposição a pagar por um diagnóstico correto (tabela em anexo e disponível no Mendeley Data), tendo como base o valor do PIB per capita brasileiro. Todos os cálculos foram realizados no Excel®.

\section{Custos}

A estimativa de custos (tabela 1) foi realizada por meio da abordagem top down, e o custo de cada estratégia foi baseado no custo unitário de cada teste. Para os testes disponíveis no SUS, os custos foram obtidos pelo SIGTAP - Sistema de Gerenciamento da Tabela e Procedimentos, Medicamentos e OPM do SUS. ${ }^{3}$ Para a ATC, foi utilizada a abordagem de microcusteio botom up para quantificação dos recursos necessários para sua realização (apêndice 1).

\section{Efetividade}

A acurácia de cada teste foi estimada com base em uma revisão da literatura realizada em 20/09/2019 com uma busca por meta-análises sobre a acurácia dos testes diagnósticos nas bases de dados MEDLINE, The Cochrane Library e Lilacs, sem restrição ao idioma. Os estudos foram selecionados de forma independente por dois revisores (P.B. e L.T.) e as discordâncias resolvidas por consenso. Se ao final da seleção de estudos houvesse mais de 1 artigo selecionado, o estudo com melhor avaliação de qualidade pelo AMSTAR ${ }^{15}$ seria utilizado. A estratégia de busca e o fluxograma de seleção das evidências estão disponíveis respectivamente nos apêndices 2 e 3 .

\section{Análise de Sensibilidade}

Para avaliar o impacto das incertezas dos valores das variáveis inseridas no modelo, foi realizada a análise de sensibilidade determinística. Foram utilizados os intervalos de confiança ou interquartil como valores máximos e mínimos de cada informação contida no modelo a partir de revisão de literatura, evidenciados na tabela 2.

\section{Considerações éticas}

Não foram realizadas pesquisas em seres humanos, nem utilizados dados confidenciais, institucionais ou pessoais. Toda a pesquisa é baseada em dados de estudos publicados em base de dados eletrônicas. Este projeto recebeu o

Tabela 1 - Custos dos testes diagnósticos em ordem crescente

\begin{tabular}{lc}
\hline Teste & Custo unitário (SIGTAP) \\
\hline Teste ergométrico & $\mathrm{R} \$ 30,00$ \\
\hline Ecocardiograma de estresse & $\mathrm{R} \$ 165,00$ \\
\hline Angiotomografia coronariana & $\mathrm{R} \$ 452,05$ \\
\hline Cintilografia miocárdica estresse e repouso & $\mathrm{R} \$ 791,59$ \\
\hline
\end{tabular}

Valores extraídos da tabela SIGTAP no ano de $2020^{3}$

Tabela 2 - Parâmetros e valores adotados no modelo de RCEI para estratégias diagnósticas de DAC

\begin{tabular}{|c|c|c|c|c|}
\hline Parâmetro & Estimativa pontual & Limite Inferior & Limite Superior & Referência \\
\hline Sensibilidade TE & 0,80 & 0,48 & 0,85 & Banerjee et al.,2012 ${ }^{16}$ \\
\hline Especificidade TE & 0,63 & 0,63 & 0,88 & Banerjee et al.,2012 ${ }^{16}$ \\
\hline Sensibilidade ECO & 0,81 & 0,70 & 0,87 & Banerjee et al.,2012 ${ }^{16}$ \\
\hline Especificidade ECO & 0,84 & 0,73 & 0,94 & Banerjee et al.,2012 ${ }^{16}$ \\
\hline Sensibilidade CM & 0,88 & 0,88 & 0,89 & Jaarsma et al., $2012{ }^{17}$ \\
\hline Especificidade CM & 0,61 & 0,59 & 0,62 & Jaarsma et al., $2012^{17}$ \\
\hline Sensibilidade ATC & 0,93 & 0,93 & 0,94 & Haase et al., 201918 \\
\hline Especificidade ATC & 0,84 & 0,84 & 0,85 & Haase et al., 201918 \\
\hline
\end{tabular}

Teste Ergométrico e EcoEstresse: (Banerjee, Newman, Van Den Bruel, \& Heneghan, 2012);

Cintilografia (Jaarsma et al., 2012); ATC (Haase et al., 2019). TE: teste ergométrico; CM: cintilografia miocárdica; ATC: angiotomografia de coronárias; ECO: ecocardiograma por estresse. 
seguinte parecer do CEP: "Trata-se de uma pesquisa de revisão sistemática da literatura que não necessita de avaliação pelo CEP". Número do parecer: 2.421.181.

\section{Resultados}

$\mathrm{Na}$ análise pela RCEI, dentre as 11 estratégias diagnósticas, foram identificadas 7 estratégias dominadas, ou seja, estratégias com maior custo e menor número de diagnósticos corretos (tabela 3).

As quatro estratégias não dominadas foram ordenadas por ordem crescente de custos, e identificou-se que a estratégia TE + ATC era menos efetiva (menor número de diagnósticos corretos) e com maior RCEI do que a estratégia ECO + ATC (tabela 4), sendo, portanto, considerada não custo-efetiva (dominância estendida).

Assim, a fronteira de eficiência foi construída com base nas três estratégias mais custo-efetivas, TE, TE+ECO e $\mathrm{ECO}+\mathrm{ATC}$ (figura 1).

Baseado na análise de sensibilidade, evidenciada no gráfico de Tornado (figura 2), os parâmetros com maior impacto nos resultados foram a sensibilidade e especificidade do teste ergométrico, o custo da ATC e a prevalência de DAC.
O ranqueamento pelo NHB permite avaliar todas as estratégias, sem a necessidade de excluir estratégias dominadas ou com dominância estendida. A tabela 5 apresenta o ranqueamento das estratégias mais custo efetivas de acordo com a variação de probabilidades préteste entre $10 \%$ e $60 \%$ em diferentes limiares de disposição a pagar por um diagnóstico correto, tendo como base o valor do PIB per capita brasileiro, que segundo última atualização do IBGE 2017 é de R\$ 31.833,50. ${ }^{19}$

\section{Discussão}

A estratégia ECO + ATC apresenta a melhor taxa de diagnóstico correto $(909,49)$, ou seja, a maior efetividade; e, portanto, maior certeza no direcionamento clínico dos pacientes, seja para a coronariografia, seja para afastar o diagnóstico. A definição das melhores estratégias de investigação de DAC obstrutiva resulta em maior certeza diagnóstica, minimizando o número de falso-negativos (perda do diagnóstico), assim como os falso-positivos (reduzindo número de cateterismos "brancos" e suas complicações). ${ }^{20,21}$ Erros diagnósticos nesse cenário estão associados a exames invasivos desnecessários, além de poderem levar a complicações como infarto agudo do miocárdio e morte devido à falta de tratamento adequado em uma doença de

Tabela 3 - Projeção das estratégias em 1000 pacientes em ordem de custo, com o número de diagnósticos corretos e identificação das dominadas

\begin{tabular}{|c|c|c|c|}
\hline Estratégia & Custo & Diagnóstico correto & Observação \\
\hline $\mathrm{TE}$ & $\mathrm{R} \$ 30.000,00$ & 681 & \\
\hline $\mathrm{TE}+\mathrm{ECO}$ & $\mathrm{R} \$ 112.335,00$ & 853 & \\
\hline ECO & $\mathrm{R} \$ 165.000,00$ & 831 & Dominada \\
\hline $\mathrm{TE}+\mathrm{ATC}$ & $\mathrm{R} \$ 255.572,95$ & 884 & \\
\hline $\mathrm{ECO}+\mathrm{ATC}$ & $\mathrm{R} \$ 325.477,75$ & 909 & \\
\hline ATC & $\mathrm{R} \$ 452.050,00$ & 871 & Dominada \\
\hline $\mathrm{ATC}+\mathrm{TE}$ & $\mathrm{R} \$ 463.732,00$ & 884 & Dominada \\
\hline ATC + ECO & $\mathrm{R} \$ 516.301,00$ & 909 & Dominada \\
\hline $\mathrm{ATC}+\mathrm{CM}$ & $\mathrm{R} \$ 760.295,15$ & 904 & Dominada \\
\hline $\mathrm{CM}$ & $\mathrm{R} \$ 791.590,00$ & 691 & Dominada \\
\hline
\end{tabular}

TE: teste ergométrico; CM: cintilografia miocárdica; ATC: angiotomografia de coronárias; ECO: ecocardiograma por estresse. Ano 2020 como referência para os valores apresentados.

Tabela 4 - Identificação de dominância estendida nas estratégias diagnósticas para DAC não dominadas

\begin{tabular}{lccc}
\hline Estratégia & Custo & Diagnóstico correto & RCEI \\
\hline TE & $\mathrm{R} \$ 30.000,00$ & 681,00 & $\mathrm{NA}$ \\
\hline TE + ECO & $\mathrm{R} \$ 112.335,00$ & 852,96 & $\mathrm{R} \$ 478,80$ \\
\hline TE + ATC & $\mathrm{R} \$ 255.572,95$ & 883,75 & $\mathrm{R} \$ 4.651,19$ \\
\hline ECO + ATC & $\mathrm{R} \$ 325.477,75$ & 909,49 & $\mathrm{R} \$ 2.716,44$ \\
\hline
\end{tabular}

TE: teste ergométrico; CM: cintilografia miocárdica; ATC: angiotomografia de coronárias; ECO: ecocardiograma por estresse. Ano 2020 como referência para os valores apresentados; RCEl: razão de custo-efetividade incremental. 


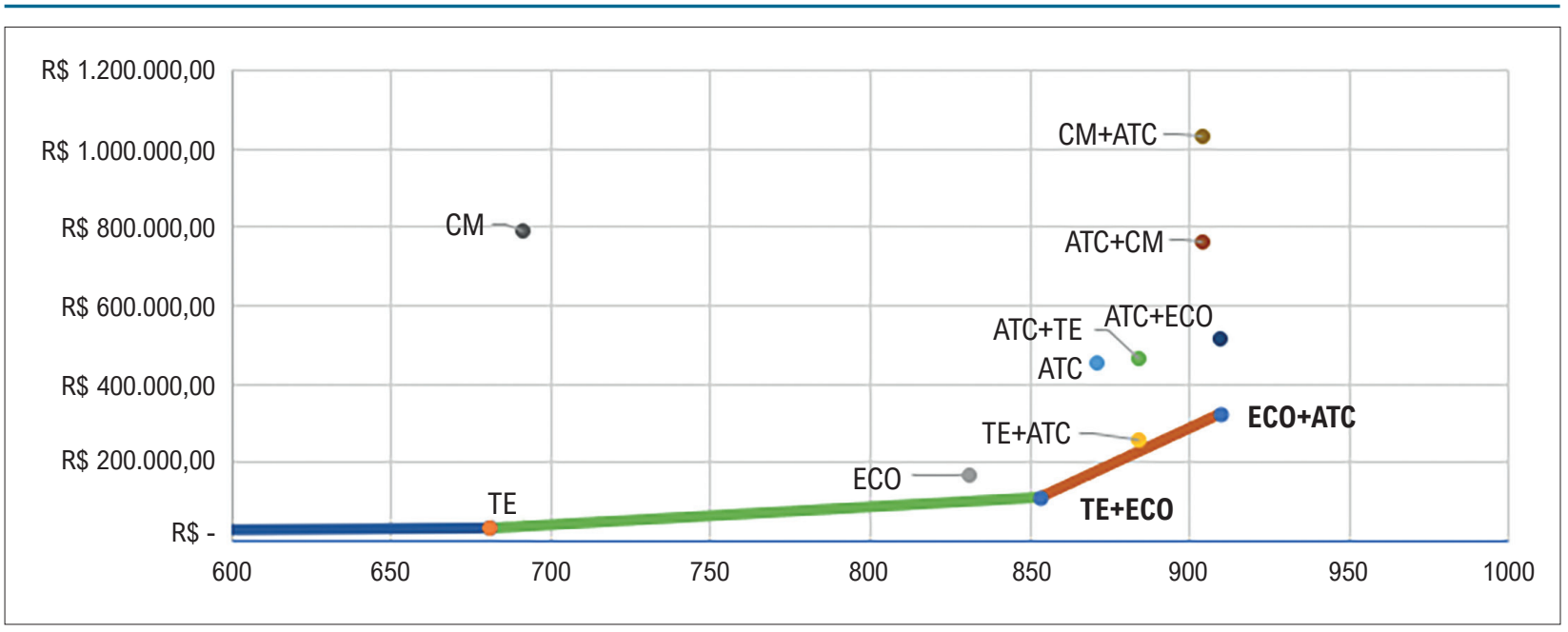

Figura 1 - Fronteira de eficiência das estratégias diagnósticas para doença coronariana (custos e número de diagnósticos corretos por mil indivíduos). TE: teste ergométrico; CM: cintilografia miocárdica; ATC: angiotomografia de coronárias; ECO: ecocardiograma por estresse.

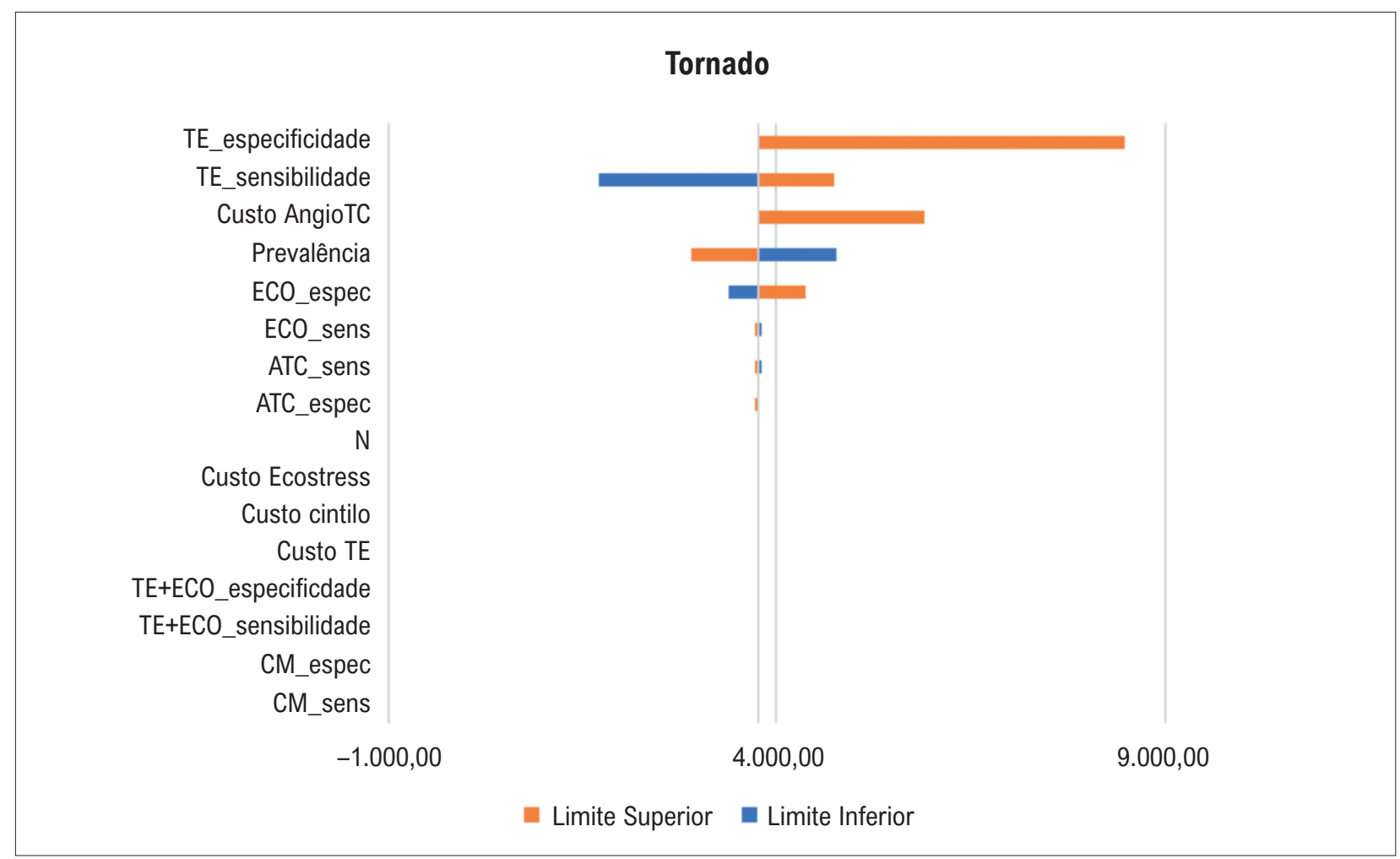

Figura 2 - Diagrama de Tornado - impacto nos valores de RCEI de cada parâmetro avaliado isoladamente em seus limites inferior e superior.

alta mortalidade. ${ }^{1,2,20}$ A acurácia dos testes diagnósticos deve ser analisada à luz de seus custos, principalmente quando consideramos uma nova tecnologia em um sistema de saúde financiado publicamente, como o SUS.

A razão de custo-efetividade incremental (RCEI) tem sido usada por agências de avaliação de tecnologia em saúde em todo o mundo para resumir os resultados de avaliações econômicas de intervenções em saúde. Mesmo em países como o Brasil, onde não existe um limiar explícito de RCEI para tomada de decisão, seu impacto nas decisões é muito relevante. No entanto, medidas sumárias alternativas baseadas no conceito de benefício líquido vêm sendo apresentadas e este é o primeiro estudo a avaliar o custo-benefício das estratégias diagnósticas para DAC por meio de uma análise de benefício líquido em saúde.

Existem distinções importantes entre o RCEI e o NHB. O RCEI necessita de comparação entre duas estratégias, 


\begin{tabular}{|c|c|c|c|c|c|c|c|}
\hline & 0,05 PIB pc & 0,1 PIB pc & 0,2 PIB pc & 0,3 PIB pc & 0,4 PIB pc & 0,5 PIB pc & 1 PIB pc \\
\hline $10 \%$ & $\mathrm{TE}+\mathrm{ECO}$ & $\mathrm{TE}+\mathrm{ECO}$ & $\mathrm{ECO}+\mathrm{ATC}$ & $\mathrm{ECO}+\mathrm{ATC}$ & $\mathrm{ECO}+\mathrm{ATC}$ & $\mathrm{ECO}+\mathrm{ATC}$ & $\mathrm{ECO}+\mathrm{ATC}$ \\
\hline $20 \%$ & $\mathrm{TE}+\mathrm{ECO}$ & $\mathrm{TE}+\mathrm{ECO}$ & $\mathrm{ECO}+\mathrm{ATC}$ & $\mathrm{ECO}+\mathrm{ATC}$ & $\mathrm{ECO}+\mathrm{ATC}$ & $\mathrm{ECO}+\mathrm{ATC}$ & $\mathrm{ECO}+\mathrm{ATC}$ \\
\hline $30 \%$ & $\mathrm{TE}+\mathrm{ECO}$ & $\mathrm{ATC}+\mathrm{TE}$ & $\mathrm{ECO}+\mathrm{ATC}$ & $\mathrm{ECO}+\mathrm{ATC}$ & $\mathrm{ECO}+\mathrm{ATC}$ & $\mathrm{ECO}+\mathrm{ATC}$ & $\mathrm{ECO}+\mathrm{ATC}$ \\
\hline $40 \%$ & $\mathrm{TE}+\mathrm{ECO}$ & $\mathrm{ATC}+\mathrm{TE}$ & $\mathrm{ECO}+\mathrm{ATC}$ & $\mathrm{ECO}+\mathrm{ATC}$ & $\mathrm{ECO}+\mathrm{ATC}$ & $\mathrm{ECO}+\mathrm{ATC}$ & $\mathrm{ECO}+\mathrm{ATC}$ \\
\hline $50 \%$ & ECO & $\mathrm{ATC}+\mathrm{TE}$ & ATC & ATC & ATC & ATC & ATC \\
\hline $60 \%$ & ECO & ECO & ATC & ATC & ATC & ATC & ATC \\
\hline
\end{tabular}

ATC: angiotomografia coronariana; CM: cintilografia miocárdica; ECO: ecocardiograma de estresse; TE: teste ergométrico; PIB: produto interno bruto.

independentemente do número total de estratégias avaliadas. No $\mathrm{NHB}$, as medidas de benefício líquido são calculadas para cada estratégia individualmente, ou seja, elimina-se a necessidade de comparação por pares e da eliminação de estratégias dominadas. É necessário um limiar estabelecido de disposição a pagar para calcular medidas de benefício líquido, mas não é necessário para calcular a RCEI, embora sem um limiar de custo-efetividade sua interpretação fique limitada. Não há um limiar de custoefetividade estabelecido no Brasil no processo de incorporação de tecnologias. Neste estudo, a estratégia mais custo-efetiva, de acordo com a fronteira de eficiência, foi a combinação de ecocardiograma de estresse seguido de angiotomografia de coronárias, dado que é a estratégia não dominada com maior valor de RCEI e dentro de um limiar.

O critério de NHB permite ranquear todas as estratégias de forma a ajudar o tomador de decisão a escolher qual estratégia seguir, com base na disponibilidade dos exames/profissionais, orçamento e disposição a pagar por diagnóstico correto. A ATC, apesar de ser o único exame não incorporado hoje ao SUS, é o exame mais prevalente entre as estratégias mais custo-efetivas, apenas não ocupando o primeiro lugar quando a disposição a pagar é inferior a 0,2 PIB per capita por diagnóstico correto. Isoladamente, desconsiderando exames sequenciais, o exame mais custo-efetivo é o ecocardiograma de estresse, até o limiar de 0,1 PIB per capita por diagnóstico correto, sendo superado pela ATC nos limiares superiores.

Ao variarmos em diferentes probabilidades pré-teste $(10 \%$ a $60 \%$ ) encontramos a ATC como teste mais custo efetivo (combinado ou não a outros métodos) em 79\% dos cenários analisados, o que está em acordo com estudos de custoefetividade da ATC realizados em países desenvolvidos ${ }^{20-23}$ além das recentes atualizações das diretrizes do Reino Unido (National Institute for Heath and Care Excellence) de 201722,24 e da diretriz da sociedade europeia (ESC). ${ }^{25}$ Atualizada em 2019, tal diretriz determina que a ATC possa ser usada como primeiro exame na avaliação de sintomas sugestivos de DAC obstrutiva, em alternativa aos exames funcionais de imagem. No Reino Unido, que possui um sistema de saúde financiado com recursos públicos (assim como o SUS no Brasil) baseado em análises de custo-efetividade para a sua realidade, foi optado pela recomendação da ATC como primeiro exame, em substituição aos exames funcionais. ${ }^{22,24}$ Essa decisão deve ser tomada baseada na realidade de cada país e cada sistema de saúde, sendo o objetivo do presente estudo aprofundar o entendimento das estratégias diagnósticas de dor torácica na realidade do SUS.

A escolha da estratégia diagnóstica deve levar em consideração não apenas a custo-efetividade para o achado da obstrução coronariana, mas também os desfechos clínicos. Grandes estudos randomizados como o PROMISE e SCOT HEART trouxeram informações principalmente sobre o prognóstico daqueles pacientes que iniciavam a investigação para DAC com a angiotomografia de coronárias. Houve maior certeza no diagnóstico e, com isso, maior introdução de terapias medicamentosas preventivas, considerando uma possibilidade real na redução de eventos como infarto a longo prazo, além de documentar menor número de coronariografias sem doença obstrutiva nesse grupo de pacientes. ${ }^{26,27}$ Nesse contexto é importante ressaltar que o grande estudo randomizado ISCHEMIA não demostrou redução de morte ou infarto no braço dos exames funcionais. ${ }^{28} \mathrm{Em}$ contrapartida, a avaliação anatômica permite o diagnóstico de aterosclerose e direciona melhor tratamento clínico, com possibilidade na redução de infarto e morte. ${ }^{26,27,29} \mathrm{~A}$ última diretriz da sociedade brasileira de cardiologia (SBC) de 2014, recomenda iniciar a investigação de DAC obstrutiva com exames funcionais, seguidos por ATC caso tais exames sejam inconclusivos ou contraindicados. ${ }^{30}$

Dentre os métodos funcionais, a CM é um dos testes mais usados no Brasil e no mundo para diagnóstico de DAC, ${ }^{31,32}$ sendo estimado que no SUS aproximadamente 54\% dos exames eletivos de medicina nuclear sejam de perfusão miocárdica. ${ }^{32}$ No entanto, as estratégias que incluíram CM foram dominadas em nosso estudo, sendo mais caras e menos efetivas.

As estratégias TE + ECO e TE + ATC apresentam percentuais de falso positivo semelhantes de 4\% (tabela 2:2 em anexo), já o percentual de falsos negativos varia de forma considerável entre as estratégias. Por exemplo, a estratégia TE + ECO apresenta taxa de falso negativo de $1,3 \%$, e a estratégia TE + ATC, com taxa de falso negativo de $0,4 \%$ (três vezes menor do que TE + ECO), dessa forma apresentando menos diagnósticos errados. Apesar de ter dominância estendida na fronteira de eficiência, observamos na análise do NHB que a estratégia TE + ATC perde para TE + ECO apenas na margem de 0,05 a 0,1 PIB per capita (tabela $\mathrm{NHB}$ em anexo). Características econômicas assimétricas entre regiões e cidades no Brasil fazem com que a disponibilidade de equipamentos e de mão de obra qualificada seja heterogênea. Por exemplo, no ano de 2019, dentre as quase seis milhões de tomografias realizadas no SUS, 51\% concentraram-se na região Sudeste e menos de $6 \%$, na região Norte. ${ }^{33} \mathrm{O}$ ranqueamento das 
opções diagnósticas apresentado neste trabalho poderá auxiliar os decisores combinando dados locais de infraestrutura, disposição a pagar e acurácia diagnóstica. A ATC é um exame menos difundido e com maquinário mais caro que o ECO, o que possivelmente a limita como primeiro exame em cenários com menor orçamento, em que o ideal seria iniciar com um exame de menor custo e complexidade, sendo os resultados positivos referenciados para exames mais caros e de maior complexidade. Sendo o teste ergométrico exame mais difundido que o ECO, há de se considerar que a estratégia TE + ATC possa ser mais exequível no sistema de saúde público brasileiro do que a estratégia ECO + ATC, apesar de pequena queda da efetividade da iniciada pelo TE comparada com a iniciada pelo ECO. Analisamos primariamente pacientes em probabilidade pré-teste de $30 \%$, por ser essa a prevalência de doença usualmente encontrada nos laboratórios diagnósticos ambulatoriais (considerada moderada-baixa). Após a realização dos testes de sensibilidade, os resultados de custo-efetividade não revelam mudanças substanciais quando variamos a probabilidade pré-teste. Além disso, foram ranqueadas as probabilidades préteste entre 10 e $60 \%$ de acordo com a disposição a pagar, sendo a ATC não encontrada como opção custo efetiva apenas quando consideramos o valor de 0,05 PIB per capita por diagnóstico correto.

Dentre as limitações desse trabalho, encontram-se divergências entre os artigos encontrados, principalmente em relação à definição de DAC obstrutiva, em que os estudos sobre ATC e CM definem como obstrução coronariana acima de 50\% à coronariografia, e o estudo que inclui teste ergométrico e ecoestresse inclui artigos com referência de DAC obstrutiva acima de $50 \%$ ou $70 \%$. Para minimizar possíveis vieses, foi utilizada estratégia de revisão sistemática além da utilização de metaanálises e análise da qualidade dos artigos. Um recente estudo de custo-efetividade com dados do SUS apresenta como principal limitação a determinação do valor da ATC como o custo pago pelo SUS por uma tomografia simples de tórax, extrapolando dados do sistema de saúde suplementar. ${ }^{34}$ Nosso trabalho tenta se aproximar ao valor real de custos da ATC (aproximadamente 3 vezes o valor da TC de tórax), uma vez que esse valor influencia majoritariamente a avaliação das estratégias comparativas. Por fim, a acurácia dos testes varia com a qualidade do equipamento e dos profissionais responsáveis. Futuros estudos poderão atestar o impacto da adoção deste fluxograma de decisão pelo seguimento

\section{Referências}

1. World Health Organization. Cardiovascular Diseases (CVDs) Factsheet [Internet]. Geneva: WHO; 2013 [cited 2018 Apr. 03]. Available from: http:// www.who.int/mediacentre/factsheets/s317/en/index.html.

2. Organização Pan-Americana de Saúde. Doenças cardiovasculares [Internet]. Geneva: World Health Organization; 2017 [cited 2018 Apr. 03]. Available from: https://www.paho.org/bra/index.php?option=com_content\&view = article\&id $=5253$ : doencas-cardiovasculares\&ltem.

3. Brasil. Ministério da Saúde. DATASUS: Tecnologia da Informação a Serviço doSUS [Internet]. Geneva: Ministério da Saúde; 2018 [cited 2020Sep. 09]. Available from: http://www2.datasus.gov.br/DATASUS/index.php?area $=0201 \& i d=1421686$.

4. Lotufo PA, Malta DC, Szwarcwald CL, Stopa SR, Vieira ML, Bensenor IM. Prevalência de Angina do Peito pelo Questionário de Rose na População Brasileira: Análise da Pesquisa Nacional de Saúde, 2013. Rev Bras Epidemiol. 2015;18(Suppl 2):123-31. doi: 10.1590/. prospectivo computando dados clínicos e econômicos dos resultados de mundo real.

\section{Conclusão}

Em cenários como o brasileiro, de restrição orçamentária e de heterogeneidade na oferta de testes diagnósticos, identificar estratégias custo-efetivas poderá orientar gestores e tomadores de decisão em saúde a gerir seus recursos de maneira mais eficiente. Utilizando conceitos atuais de avaliação de tecnologias em saúde, este estudo fornece um ranqueamento para a tomada de decisão sobre qual estratégia diagnóstica utilizar, em uma população com risco pré-teste intermediário para DAC. Adotando-se uma estimativa factível de custos para a ATC, conclui-se que o impacto da inclusão desta ao rol do arsenal diagnóstico representaria uma estratégia custo-efetiva na maioria dos cenários avaliados com amplas variações na disposição a pagar.

\section{Contribuição dos autores}

Concepção e desenho da pesquisa e Revisão crítica do manuscrito quanto ao conteúdo intelectual importante: Carmo PB, Rey HCV, Gottlieb I; Obtenção de dados: Carmo PB, Trocado L; Análise e interpretação dos dados: Carmo PB, Magliano C, Rey HCV, Camargo G, Gottlieb I; Análise estatística: Magliano C; Redação do manuscrito: Carmo PB, Magliano C, Camargo G, Gottlieb I.

\section{Potencial conflito de interesse}

Dr. Gabriel C. Camargo - Trabalha em empresa privada com a realização de ATC. Dr. Ilan Gottlieb - Trabalha em empresa privada com a realização de ATC.

\section{Fontes de financiamento}

O presente estudo não teve fontes de financiamento externas.

\section{Vinculação acadêmica}

Este artigo é parte de dissertação de mestrado de Patricia Bastos do Carmo pelo Instituto Nacional de Cardiologia.

5. Siqueira ASE, Siqueira-Filho AG, Land MGP. Análise do Impacto Econômico das Doenças Cardiovasculares nos Últimos Cinco Anos no Brasil. Arq Bras Cardiol. 2017;109(1):39-46. doi: 10.5935/abc.20170068.

6. Rossato G, Quadros AS, Sarmento-Leite R, Gottschall CAM. Análise das Complicações Hospitalares Relacionadas ao Cateterismo Cardíaco. Rev Bras Cardiol Invasiva. 2007;15(1):44-51. doi: 10.1590/S217983972007000100010 .

7. Chandrasekar B, Doucet S, Bilodeau L, Crepeau J, deGuise P, Gregoire J, et al. Complications of Cardiac Catheterization in the Current Era: A Single-Center Experience. Catheter Cardiovasc Interv. 2001;52(3):289-95. doi: 10.1002/ ccd.1067.

8. Patel MR, Peterson ED, Dai D, Brennan JM, Redberg RF, Anderson HV, et al. Low Diagnostic Yield of Elective Coronary Angiography. N Engl J Med. 2010;362(10):886-95. doi: 10.1056/NEJMoa0907272. 
9. Elias P, Barbosa P, Santos VS, Cruz D, Alcântara RW, Silva FAV, et al. Adequacy of Elective Coronary Angiography Indication for the Diagnosis of Coronary Artery Disease in the Brazilian Public Health System. Rev Bras Cardiol Invasiva. 2017;25(1-4):2-6. do

10. George RT, Arbab-Zadeh A, Miller JM, Vavere AL, Bengel FM, Lardo AC, et al. Computed Tomography Myocardial Perfusion Imaging with 320-row Detector Computed Tomography Accurately Detects Myocardial Ischemia in Patients with Obstructive Coronary Artery Dise.

11. Budoff MJ, Li D, Kazerooni EA, Thomas GS, Mieres JH, Shaw LJ. Diagnostic Accuracy of Noninvasive 64-row Computed Tomographic Coronary Angiography (CCTA) Compared with Myocardial Perfusion Imaging (MPI): The PICTURE Study, A Prospective Multicenter Trial.

12. Miller JM, Rochitte CE, Dewey M, Arbab-Zadeh A, Niinuma H, Gottlieb I, et al. Diagnostic Performance of Coronary Angiography by 64-row CT. N Eng J Med. 2008;359(22):2324-36. doi: 10.1056/NEJMoa0806576.

13. Sørgaard MH, Kofoed KF, Linde J], George RT, Rochitte CE, Feuchtner G, et al. Diagnostic Accuracy of Static CT Perfusion for the Detection of Myocardial Ischemia. A Systematic Review and Meta-Analysis. J Cardiovasc Comput Tomogr. 2016;10(6):450-7. doi: 10.

14. Paulden M. Calculating and Interpreting ICERs and Net Benefit. Pharmacoeconomics. 2020;38(8):785-807. doi: 10.1007/s40273-02000914-6.

15. Shea BJ, Reeves BC, Wells G, Thuku M, Hamel C, Moran J, et al. AMSTAR 2: A Critical Appraisal Tool for Systematic Reviews that Include Randomised or Non-Randomised Studies of Healthcare Interventions, or Both. BMJ. 2017;358:4008. doi: 10.1136/bmj.j4008.

16. Banerjee A, Newman DR, Van Den Bruel A, Heneghan C. Diagnostic Accuracy of Exercise Stress Testing for Coronary Artery Disease: A Systematic Review and Meta-Analysis of Prospective Studies. Int J Clin Pract. 2012;66(5):477-9. doi: 10.1111/j.1742-1241.2012.

17. Jaarsma C, Leiner T, Bekkers SC, Crijns HJ, Wildberger JE, Nagel E, et al. Diagnostic Performance of Noninvasive Myocardial Perfusion Imaging Using Single-Photon Emission Computed Tomography, Cardiac Magnetic Resonance, and Positron Emission Tomography Im.

18. Haase R, Schlattmann P, Gueret P, Andreini D, Pontone G, Alkadhi H, et al. Diagnosis of Obstructive Coronary Artery Disease using Computed Tomography Angiography in Patients with Stable Chest Pain Depending on Clinical Probability and in Clinically Import.

19. Instituto Brasileiro de Geografia e Estatística. O que é PIB? [Internet]. Rio de Janeiro: IBGE; c2021 [cited 2020 Sep 24]. Available from: https://www. ibge.gov.br/explica/pib.php.

20. Ferreira AM, Marques H, Gonçalves PA, Cardim N. Cost-Effectiveness of Different Diagnostic Strategies in Suspected Stable Coronary Artery Disease in Portugal. Arq Bras Cardiol. 2014;102(4):391-402. doi: 10.5935/ abc. 20140042 .

21. Genders TS, Steyerberg EW, Alkadhi H, Leschka S, Desbiolles L, Nieman K, et al. A Clinical Prediction Rule for the Diagnosis of Coronary Artery Disease:
Validation, Updating, and Extension. Eur HeartJ. 2011;32(11):1316-30. doi: 10.1093/eurheartj/ehr014.

22. Adamson PD, Hunter A, Williams MC, Shah ASV, McAllister DA, Pawade TA, et al. Diagnostic and Prognostic Benefits of Computed Tomography Coronary Angiography using the 2016 National Institute for Health and Care Excellence Guidance within a Randomised Tria.

23. Lee SP, Jang EJ, Kim YJ, Cha MJ, Park SY, Song HJ, et al. Cost-Effectiveness of Coronary CT Angiography in Patients with Chest Pain: Comparison with Myocardial Single Photon Emission Tomography. J Cardiovasc Comput Tomogr. 2015;9(5):428-37. doi: 10.1016/j

24. Moss AJ, Williams MC, Newby DE, Nicol ED. The Updated NICE Guidelines: Cardiac CT as the First-Line Test for Coronary Artery Disease. Curr Cardiovasc Imaging Rep. 2017;10(5):15. doi: 10.1007/s12410-017-9412-6.

25. Knuuti J, Wijns W, Saraste A, Capodanno D, Barbato E, Funck-Brentano C, et al. 2019 ESC Guidelines for the Diagnosis and Management of Chronic Coronary Syndromes. 2019;1-71. doi: 10.1093/eurheartj/ehz425.

26. Douglas PS, Hoffmann U, Patel MR, Mark DB, Al-Khalidi HR, Cavanaugh B, et al. Outcomes of Anatomical Versus Functional Testing for Coronary Artery Disease. N Engl J Med. 2015;372(14):1291-300. doi: 10.1056/ NEJMoa1415516.

27. SCOT-HEART Investigators. CT Coronary Angiography in Patients with Suspected Angina Due to Coronary Heart Disease (SCOT-HEART): An OpenLabel, Parallel-Group, Multicentre Trial. Lancet. 2015;385(9985):2383-91. doi: 10.1016/S0140-6736(15)60291-4

28. Maron DJ, Hochman JS, Reynolds HR, Bangalore S, O'Brien SM, Boden WE, et al. Initial Invasive or Conservative Strategy for Stable Coronary Disease. N Engl J Med. 2020;382(15):1395-407. doi: 10.1056/NEJMoa1915922.

29. Newby DE, Adamson PD, Berry C, Boon NA, Dweck MR, Flather M, et al. Coronary CT Angiography and 5-Year Risk of Myocardial Infarction. N Engl J Med. 2018;379(10):924-33. doi: 10.1056/NEJMoa1805971.

30. Cesar LA, Ferreira JF, Armaganijan D, Gowdak LH, Mansur AP, Bodanese LC, et al. Guideline for Stable Coronary Artery Disease. Arq Bras Cardiol. 2014;103(2 Suppl 2):1-56. doi: 10.5935/abc.2014s004.

31. Pozzo L, Coura Filho G, Osso JA Jr, Squair PL. O SUS na Medicina Nuclear do Brasil: Avaliação e Comparação dos Dados Fornecidos pelo Datasus e CNEN. Radiol Bras. 2014;47(3):141-8. doi: 10.1590/0100-3984.2013.1906.

32. Santos MA, Santos MS, Tura BR, Félix R, Brito ASX, De Lorenzo A. Budget Impact of Applying Appropriateness Criteria for Myocardial Perfusion Scintigraphy: The Perspective of a Developing Country. J Nucl Cardiol. 2016:23(5):1160-5. doi: 10.1007/s12350-016-

33. Brasil. Ministerio da Saúde. Produção Ambulatorial do SUS Brasil: por local de atendimento [Internet]. Brasília: DATASUS; 2018 [cited 2020 Dec 05]. Available from: http://tabnet.datasus.gov.br/cgi/tabcgi.exe?sia/cnv/qauf.def.

34. Bertoldi EG, Stella SF, Rohde LE, Polanczyk CA. Long-Term Cost-Effectiveness of Diagnostic Tests for Assessing Stable Chest Pain: Modeled Analysis of Anatomical and Functional Strategies. Clin Cardiol. 2016;39(5):249-56. doi: $10.1002 / \mathrm{clc} .22532$
*Material suplementar
Para informação adicional, por favor, clique aqui. 